\title{
EXPRESSIVE REASONING WITH HORN RULES AND FUZZY DESCRIPTION LOGICS
}

\author{
Theofilos Mailis and Stefanos Kollias \\ Department of Electrical and Computer Engineering, \\ National Technical University of Athens, \\ Zographou 15780, Greece
}

\begin{abstract}
This paper describes fuzzy CARIN, a knowledge representation language combining fuzzy description logics with Horn Rules. Fuzzy CARIN integrates the management of fuzzy logic into the non recursive CARIN system. It provides a sound and complete algorithm

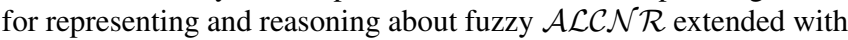
non recursive Horn Rules. Such an extension is most useful in realistic applications dealing with uncertainty and imprecision, such as multimedia processing and medical applications. Additionally, it provides the ability of answering to union of conjunctive queries, which is a novelty not previously addressed by fuzzy DL systems.
\end{abstract}

\section{INTRODUCTION}

Over the last two decades fragments of first order logic, called Description Logics (DLs) [1], have been brought into focus by the Artificial Intelligence community. DLs well founded semantics and great expressivity has enforced their utilization in numerous domains, such as multimedia [2] and medical applications, as knowledge representation and reasoning languages. More importantly DLs provide the formal foundation for the standard web ontology language OWL [3] which is a milestone for the Semantic Web [4].

DLs main asset, their class-based knowledge representation formalism, also sets a limit to their expressive power as they are incapable of providing complex descriptions about role predicates. Expressive DLs such as $\mathcal{S H O I} \mathcal{Q}$ are incapable of expressing even a simple composition between roles. For this reason, as visualized in the Semantic Web stack diagram, there is a need for integrating DLs with rules. A natural choice for such integration would be classes of rule languages originating from logic programming and non-monotonic reasoning [5].

In [5], the "cream" of systems combining rules and DLs is presented. Systems such as DLP [6], SWRL [7], $\mathcal{A} \mathcal{L}$-log [8], $F$-logic [9] and CARIN [10] present different approximations for intergrading DLs with rules. These are divided into the hybrid systems, where there is a distinction between the predicates in the rule and the DL part, and the homogeneous where there is no such distinction. CARIN is such an hybrid system that combines the DL $\mathcal{A L C N \mathcal { R }}$ with Horn Rules and through its existential entailment algorithm offers a sound and complete inference procedure for non-recursive knowledge bases, can answer to arbitrary conjunctive queries and provides an algo-

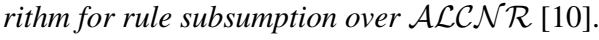

Though CARIN offers great expressivity in order to represent a fragment of our universe, it is incapable of encoding knowledge with some degree of uncertainty and imprecision. Uncertainty emerges from our lack of knowledge about a certain fact e.g. we assume that the black dot in the background of a picture is a lion, while imprecision refers to the intrinsic inability to strictly classify a fact or a state of an object e.g. a half-empty glass of water can neither be characterized as full, nor as empty.

Fuzzy logic is a means to represent knowledge containing uncertainty and imprecision. Several systems, such as fuzzy $f_{K D}-$ $\mathcal{A L C}[11]$, fuzzy $f_{K D}-\mathcal{S I}[12,13], f_{K D}-\mathcal{S H} \mathcal{I} \mathcal{N}[14]$, have been proposed for combining fuzzy logic with description logics. Based on these systems we propose fuzzy CARIN, which is an extension of non-recursive CARIN, in order to represent uncertainty and imprecision. The need for fuzzy extensions of systems combining DLs with rules is most evident in multimedia applications.

\section{THE LANGUAGE OF FUZZY CARIN}

A fuzzy CARIN knowledge base $K$ is composed of three components $K=\langle\mathcal{T}, \mathcal{H}, \mathcal{A}\rangle$, a DL terminology component $\mathcal{T}$ also called a TBox, a Horn Rules component $\mathcal{H}$ and a ground facts component $\mathcal{A}$ also called an ABox. In the syntax that we propose, we consider that fuzziness exists only in the ground facts component. For example we can assert that the weather is cloudy with a degree greater or equal than 0.6: (weather: cloudy) $\geq 0.6$.

Fuzzy CARIN's primitive elements are a set of individuals I, an alphabet of concept names $\mathbf{C}$, role names $\mathbf{R}$ and ordinary predicate names $\mathbf{Q}$. Elements of $\mathbf{I}$ represent the objects in our universe, while $\mathbf{C}$ and $\mathbf{R}$ correspond to unary and binary fuzzy relationships between individuals in $\mathbf{I}$. Elements of $\mathbf{Q}$ correspond to relationships, between individuals, of any arity.

Terminological component in fuzzy CARIN: The fuzzy CARIN terminological component $\mathcal{T}$ has the same syntax as the crisp. Complex concepts are built from concept and role names using the constructors of $\mathcal{A L C \mathcal { N }}$ as described in Equation 1 where $A$ is a concept name, $C$ and $D$ are concept descriptions, $R$ is a role definition described in Equation 2 and $P_{1}, \ldots, P_{k}$ are role names in $\mathbf{R}$.

$$
\begin{aligned}
& C, D \longrightarrow A|\top| \perp|C \sqcap D| C \sqcup D|\neg C| \forall R . C \mid \\
& \exists R . C|(\geq m R)|(\leq m R) \\
& R:=P_{1} \sqcap \ldots \sqcap P_{k}
\end{aligned}
$$

The TBox contains a set of concept definitions of the form $A:=$ $D$ and concept inclusions of the form $C \sqsubseteq D$

Horn Rules in fuzzy CARIN: The Horn rule component $\mathcal{H}$ of a fuzzy CARIN knowledge base $K$ contains a set of Horn Rules that are logical sentences of the form:

$$
p_{1}\left(\bar{X}_{1}\right) \wedge \ldots \wedge p_{k}\left(\bar{X}_{k}\right) \Rightarrow q(\bar{Y})
$$

where $\bar{X}_{1}, \cdots, \bar{X}_{k}$ and $\bar{Y}$ are tuples of variables and individuals and $p_{1}, \cdots p_{k}$ may be concept names, roles or ordinary predicates 


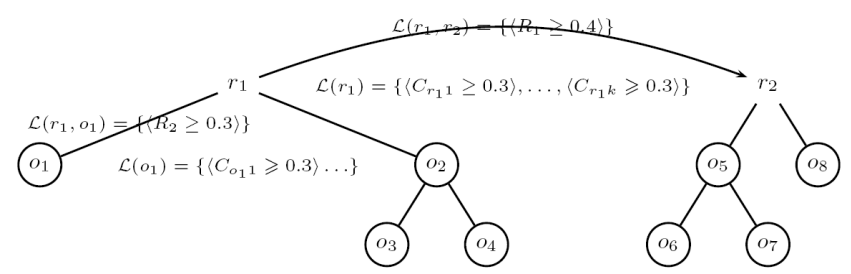

Fig. 1. An ALCNR completion forest

while $q$ is always an ordinary predicate. The antecedent of a Horn rule is called its body and the consequent is called its head.

Ground fact component: The ground fact component $\mathcal{A}$ of a fuzzy CARIN knowledge base contains a set of fuzzy assertions as shown in table 1:

Table 1. Fuzzy CARIN assertions

\section{$(a: C) \bowtie n,(\langle a, b\rangle: P) \triangleright n,(\bar{a}: p) \triangleright n$}

where $a, b \in \mathbf{I}, \bar{a}$ is a tuple of individuals,

$\bowtie \in\{\geqslant,>, \leqslant,<\}, \triangleright \in\{\geqslant,>\}$,

$C$ is a concept description, $P$ is a role name, $p$ is an ordinary

predicate of the same arity as $\bar{a}$ and $n \in[0,1]$

Intuitively a fuzzy assertion of the form (weather : cloudy) $\geqslant$ 0.5 means that the weather is cloudy with a degree at least equal to 0.5 . We call assertions defined by $\geqslant,>$ positive assertions, denoted with $\triangleright$, while those defined by $\leqslant,<$ negative assertions, denoted with $\triangleleft$. $\bowtie$ stands for any type of inequality. In fuzzy CARIN, we consider only positive role assertion, since negative assertions would imply the existence of role negation and disjunction of roles in

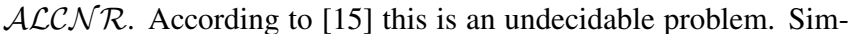
ilarly for ordinary predicates we use only positive assertions since negation cannot be expressed in simple Horn Rules. Finally negative concept assertions can be reduced to positive ones with the use of negation i.e. $(a: C)<n$ can be reduced to $(a: \neg C) \geq 1-n$.

\section{REASONING IN FUZZY CARIN}

The reasoning procedure of Fuzzy Carin is performed in two steps. In the first step a set of completion forests corresponding to the DL part of our Knowledge Base is created. The creation of each completion forest, such as presented in Figure 1, is based on the tableaux algorithm for fuzzy- $\mathcal{A} \mathcal{L} \mathcal{C N} \mathcal{R}$.

In the second step a graph matching algorithm populates the ordinary predicates in the head of each Horn Rule. This procedure can be easily done by a graph matching algorithm since in our knowledge we have only acyclic Horn Rules. We consider that an individual $a$ is an instance of a concept $C$ to some degree greater or equal than $n$, if in each completion forest $\mathcal{F}$ we have that $C(a) \geq n^{\prime}$, where $n^{\prime} \geq n$. The same will apply for a pair of individuals and a Role, or a tuple of individuals and an Ordinary Predicate, only that in the last case we consider that the knowledge deriving from each completion forest $\mathcal{F}$ is augmented according to the Horn Rules.

\section{CONCLUSIONS}

In this paper we have presented the fuzzy Carin algorithm which combines fuzzy DLs with Horn Rules. The expressiveness of our algorithm could be proved most useful especially in multimedia applications. A more detailed description of our algorithm along with some examples is described in [16].

\section{REFERENCES}

[1] Baader, F., Calvanese, D., McGuinness, D.L., Nardi, D., PatelSchneider, P.F., eds.: The Description Logic Handbook: Theory, Implementation, and Applications. In Baader, F., Calvanese, D., McGuinness, D.L., Nardi, D., Patel-Schneider, P.F., eds.: Description Logic Handbook, Cambridge University Press (2003)

[2] Meghini, C., Sebastiani, F., Straccia, U.: A model of multimedia information retrieval. J. ACM 48 (2001) 909-970

[3] Herman, I., Hendler, J.: Web ontology language (OWL). technical report (2004) http://www.w3.org/2004/OWL/.

[4] Herman, I.: Semantic web. technical report (2007) http://www.w3.org/2001/sw/.

[5] Antoniou, G., Damasio, C.V., Grosof, B., Horrocks, I., Kifer, M., Maluszynski, J., Patel-Schneider, P.F.: Combining rules and ontologies. a survey (2006)

[6] Grosof, B.N., Horrocks, I., Volz, R., Decker, S.: Description logic programs: combining logic programs with description logic. In: WWW. (2003) 48-57

[7] Horrocks, I., Patel-Schneider, P.F.: A proposal for an owl rules language. In Feldman, S.I., Uretsky, M., Najork, M., Wills, C.E., eds.: WWW, ACM (2004) 723-731

[8] Donini, F.M., Lenzerini, M., Nardi, D., Schaerf, A.: Al-log: Integrating datalog and description logics. J. Intell. Inf. Syst. 10 (1998) 227-252

[9] Kifer, M., Lausen, G., Wu, J.: Logical foundations of objectoriented and frame-based languages. J. ACM 42 (1995) 741843

[10] Levy, A.Y., Rousset, M.C.: Combining horn rules and description logics in carin. Artif. Intell. 104 (1998) 165-209

[11] Straccia, U.: Reasoning within fuzzy description logics. J. Artif. Intell. Res. (JAIR) 14 (2001) 137-166

[12] Stoilos, G., Stamou, G., Tzouvaras, V., Pan, J., Horrocks, I.: A fuzzy description logic for multimedia knowledge representation, Proc. of the International Workshop on Multimedia and the Semantic Web (2005)

[13] Stoilos, G., Stamou, G., Pan, J., Tzouvaras, V., Horrocks, I.: Reasoning with very expressive fuzzy description logics. Journal of Artificial Intelligence Research, 30, 8, 273-320 (2007)

[14] Stoilos, G., Stamou, G.B., Tzouvaras, V., Pan, J.Z., Horrocks, I.: The fuzzy description logic f-shin. In da Costa, P.C.G., Laskey, K.B., Laskey, K.J., Pool, M., eds.: ISWC-URSW. (2005) 67-76

[15] Tobies, S.: Complexity results and practical algorithms for logics in knowledge representation (2001)

[16] Mailis, T.P., Stoilos, G., Stamou, G.B.: Expressive reasoning with horn rules and fuzzy description logics. In Marchiori, M., Pan, J.Z., de Sainte Marie, C., eds.: RR. Volume 4524 of Lecture Notes in Computer Science., Springer (2007) 43-57 